\title{
A Data-Driven Soft Sensor for Swarm Motion Speed Prediction using Ensemble Learning Methods
}

\author{
Belkacem Khaldi, Fouzi Harrou, Member, IEEE, Sidi Mohammed Benslimane and Ying Sun
}

\begin{abstract}
Machine Learning (ML) for swarm motion prediction is a relatively unexplored area that could help sustain and monitor daily swarm robotics collective tasks. This paper focuses on a specific application of swarm robotics which is pattern formation, to demonstrate the ability of Ensemble Learning (EL) approaches to predict the motion speed of swarm robots. Specifically, the boosted trees (BST) and bagged trees (BT) algorithms are introduced to predict the motion speed of a swarm of miniature two-wheels differential driver mobile robots performing a circle-formation via the viscoelastic control model. This choice's motivation is due to EL-based models' ability to improve the performance of ML models by combining multiple learners versus single regressors. Both BST and BT algorithms' performances are compared to ten commonly known prediction models based on Support Vector Regressors (SVRs) and Gaussian Process Regressors (GPRs) with different kernel functions. Using simulated measurements recorded every 0.1 second from the robots' sensors, we demonstrate the effectiveness of the developed methods over conventional ML models (SVR and GPR) in a free/non-free obstacles environment. Results showed that the BST and BT regression models reached the highest prediction performance with fully and partially connected swarms and even when involving different swarm sizes.
\end{abstract}

Index Terms-Swarm Robotics, Swarm Motion Speed Prediction, Ensemble Learning, boosted trees, bagged trees, Support Vector Regressors, Gaussian Process Regressors

\section{INTRODUCTION}

$\mathbf{S}$ WARM robotics is considered to be the systematic application of the theory behind the intelligence of natural swarm systems in controlling a large number of simple autonomous robots [1]-[3]. Therefore, it is a distributed solution approach to the problems beyond the capabilities of individual robots. Similar to their counterparts in the biological swarm ecosystem [4], robot swarm systems are characterized by a set of key features such as flexibility, robustness, and scalability [5]. These make them very practical and useful in many application scenarios that involve warehouse automation, environmental monitoring, search and rescue, inspection and exploration, and object transportation and manipulation [6].

Despite the benefits that may be brought when using such robotics systems; however, the controllers at the microscopic levels and which led to the desired collective behaviors at the macroscopic levels are still challenging to design and predict [7]. Predicting robot swarm behaviors at microscopic or macroscopic levels plays a vital role in designing pre-

Belkacem Khaldi and Sidi Mohammed Benslimane are with the Laboratoire de Recherche en Informatique de Sidi Bel Abbes (LabRI-SBA) Sidi Bel Abess, Algeria. They are also with the École Supérieure en Informatique 8 Mai 1945, Sidi Bel-Abbés, Algeria (email:b.khaldi@esisba.dz, s.benslimane@esi-sba.dz)

Fouzi Harrou and Ying Sun are with the King Abdullah University of Science and Technology (KAUST), Computer, Electrical and Mathematical Sciences and Engineering (CEMSE) Division, Thuwal, 23955-6900, Saudi Arabia (email: fouzi.harrou@kaust.edu.sa, ying.sun@kaust.edu.sa). cise controllers for individual robots and understanding the swarms' full behavior leading to their design [8].

Generally speaking, predictive models at the microscopic level focus practically on capturing the dynamics of swarm components and their interactions and their environment [2], [8]. Although with these models, precise behavior predictions are achievable. However, due to the apparent tracking of individuals and their interactions, they are not sufficiently scalable when the size of the swarm is sizeable [9]. On the other hand, predictive models at the macroscopic level consider the swarm as a whole and thus provide a high-level abstraction of the swarm dynamics, resulting in their high adaptability to swarms with large sizes [8], [10].

Many of the macroscopic predictive models of collective swarm behaviors are part of a wide range of mathematical models. Most of them use sets of differential equations to track swarm properties associated with the swarm collective behaviour [11], [12]. Despite the valuable insights that these models have brought to understand swarm behaviors, however, these models suffer from the likelihood of losing the aptitude to seizure outliers hidden in the process [11], [13].

Meanwhile, a wide variety of algorithms, particularly appropriate to prediction problems, have been developed by the computer scientists community. This includes support vector regressions (SVRs), Gaussian Process Regressions (GPRs) and Ensemble Learning (EL) models. [14], [15]. These machine learning (ML) methods have been widely used for monitoring and learning purposes in many domains of application. For example, SVR modeling techniques were applied for bearing 
health monitoring of stationary/nonstationary vibration signals [16] and for monitoring pollutant emissions from a biomass combustion process [17]. The GPR modelling technique was successfully applied in an indoor localization framework for trajectory learning purposes using crowdsourced measurements [18]. When addressing acoustic tomography reconstruction, GPR also provided a significant improvement in the reconstruction quality of the temperature distribution measurement [19]. In agriculture, EL techniques such as boosting and bagging algorithms have been used successfully to classify plants infected with Citrus Tristeza virus from healthy ones [20]. Despite the successful applications of these ML methods in other domains of application [21]. However, to the best of our knowledge, they are less often used in swarm robotics research field, probably because such ML algorithms are considered less interpretable in this area of study.

On the other hand, collective motion is one of the collective tasks that have been widely studied in the swarm robotics literature [22]-[28]. It is a fundamental operation for the cohesive movement of swarm robotics systems from one place to another. Furthermore, the motion speed of a group of robots can be crucial in such behavior and to be able to accurately predict the swarm motion speed is even more vital to involve swarm robotics systems in daily collective tasks sustainably.

In this study, we seek to open a new direction in the application of ML predictive modelling techniques in the swarm robotics area of research. More precisely, we are interested in predicting the swarm motion speed of a group of miniature robots collectively moving in a circle formation task [29]. In particular, we aim to study the prediction accuracy of EL techniques in enhancing the prediction of the swarm motion speed. This is mainly motivated by the ability of EL-based models to improve the performance of ML models by combining multiple learners versus single learners, such as SVRs and GPRs. Also, prediction error variances can be reduced using EL-based models while achieving a weak bias. The EL algorithms chosen to be used in this study are the boosted trees (BST) and the bagged Trees (BT) algorithms. These regression approaches can reach high regression performance and minimize overall prediction error by merging multiple single trees. Moreover, they allow optimizing computation time and prediction accuracy when compared to individual learners. For the best of our knowledge, these EL algorithms have not yet been applied by the swarm robotics community.

In this work, the swarm motion speed is driven by an artificial physical-based circle formation controller [29] implemented on a group of 8 differential driver mobile robots (See Subsection III-A and III-B). The EL-based models, specifically BST and the BT algorithms, proposed in this study in Subsection III-D are based only on two factors: the length and the angle of the total virtual physical force governing the motion of each robot in the swarm and which leading to the accomplishment of the circle formation task. To testify the robustness of the EL prediction strategies, three experimental simulations that incorporates: a fully connected swarm (See Subsection IV-C), a partially connected swarm (See Subsection IV-D), as well as the robustness of the approaches towards swarm scalability (See Subsection IV-E) are conducted. The datasets representing these case studies are used to build and test the proposed models. All are collected every 0.1 second from the sensors/actuators of the robots while performing the assigned task using the ARGoS simulator [30] (See Subsection III-C). The conducted experiments discussed in Section IV and which compare the prediction quality of the EL-techniques against a variety of SVRs and GPRs, clearly testify the superiority of BST and BT in all case studies. However, in a non-free obstacles scenario, the prediction performance of the BT algorithm is slightly better than that of the cubic Gaussian SVR and the rational quadratic GPR when using a fully connected swarm.

The remaining sections of the paper are arranged as follows. Section II highlights literature reviews on the related works and Section V pointed some concluding remarks and future works.

\section{RELATED WORKS}

\section{A. Microscopic based predictive models}

Predictive behaviors in this type of model are mostly performed using stochastic events to represent the behavior of each robot and are therefore not scalable to large systems [11]. For example, a stochastic model based on a Bio-PEPA has been successfully applied to predict consensus when solving a collective decision-making problem [31]. Another stochastic model based on a sequence of probabilistic events was used in a swarm aggregation mission to predict two aggregation variables: the largest aggregation ratio and the largest aggregation size [32]. To enable continuous swarm operations without relying on frequent robot battery recharging, Arvin et al. [33] applied a probabilistic stochastic model where the reduction in robot speeds during charging time was correctly predicted. In another work, Arvin et al. [34] investigated the aggregation behavior of pheromone-guided swarm robots using a probabilistic finite state machine to predict the location of aggregation and aggregation time. Lancaster Jr [11] predicted the location of the swarms using a probabilistic graph approach, which can capture the local ratio and swarms movement direction in different dimensions of worlds. While addressing a collective transport mission, Rubenstein et al. [35] predicts via a physics-based model several features including rotation and speed of transported objects, the optimal number of agents, swarm scalability and swarm performance. In a swarm inspection mission, Prorok et al. [36] followed a stochastic Markov chain methodology to accurately predict a distributed swarm robotics' spatial distribution over time and how often such system visit inspected sites.

\section{B. Macroscopic based predictive models}

By taking inspiration from a well-known decision-making process in selecting nest sites in honeybees, Reina et al. [37] derived a solution to predict the proportion of agents involved in the collective decision-making behavior within a swarm agent system. Valentini et al. [38] designed a weighted voting model to predict consensus time and decision precision using a set of ordinary differential equations. In other works, Valentini et al. [39], [40] proposed a new decision-making prediction 
strategy in which they investigated the trade-off between speed and accuracy using a real robotic swarm platform consisting of 100 kilobots. Also, Hamann et al. [41] and Goswami and Hamann [42] successfully predicted the expected swarm collective decision-making behavior using a stochastic differential based mathematical model.

In a multi-robotics task allocation mission, Zhou et al. [43] generalized a mathematical model based on a set of statistical concepts and 2D Markov process to predict the steady-state division of labor. The authors validated their model by verifying the generalized mathematical model with experimental simulations of a large number of robots. For addressing the same mission, Guerrero et al. [44] used a fuzzy Markov chain model to predict the incremental steps required to achieve a steady-state within a multi-robot swarm system.

By using a mathematical model based on birth-death activities, Khaluf et al. [45] estimated the energy consumption, the expected costs-rewards, and the expected amount of performance work in a swarm robotics area coverage scenario. Also, in a multi-robotic stigmergic coverage mission, RanjbarSahraei et al. [46] were able to estimate the convergence time using a Markov chain based macroscopic model.

In other missions scenarios, Brambilla et al. [7] presented a property-driven methodology based on a continuous-time Markov chain applied in a framework consisting of prescriptive modeling and model verification steps to predict the number of robots engaged in aggregation and foraging missions. Hamann et al. [47] proposed a general mathematical model for predicting swarm robustness and swarm performance in various swarm robot scenarios, including collective decisionmaking and foraging emerging taxis, and aggregation. Pitonakova et al. [13] suggested in the context of a foraging task with a swarm robotics system a framework called InformationCost-Reward (ICR). In ICR, the swarm performance is predicted using several measures such as rate of information gain, scouting effectiveness, and costs of uncertainty, displacement, and misinformation.

\section{Materials and Methods}

In this section, we introduce the different materials and methods used in this work. We specifically present the robotics platform. Then we briefly review the circle formation control model used in [29], [48]. Next, we explain how we collect measurements data related to the formation controller and finally we introduce the main ensemble learning methods applied for swarm motion prediction.

\section{A. Robotics Platform}

The robotics platform used in this study is the foot-bot platform: a miniature two wheels deferential driver mobile robot, which was specifically designed to conduct research studies on swarm robotics in the context of the Swarmanoid Project ${ }^{1}$. A schematic illustration of the foot-bot robot is shown in Fig.1. The foot-bot can move at a maximum speed of $30 \mathrm{~cm} / \mathrm{s}$ using two-wheel motors, each paired with a wheel and a rubber

\footnotetext{
${ }^{1}$ www.e-swarm.org
}

track. It has a number of infrared (IR) sensors on the main printed circuits distributed as follows: 24 IRs for obstacles and proximities detection and 8 IRs for ground detection. The footbot is also equipped with an omnidirectional camera for visualcolored perceiving and 13 RGB LEDs for visual-colored based communications with other foot-bots. Additionally, It comes with the range and bearing ( $\mathrm{RaB})$ communication module, which can be used for inter-robot communications purposes. This module is complemented by an IR/Radio Frequency (RF) communication technology: a combination of 16 IRs Leds transmitters and 8 IRs photodetectors. The RaB module specificity allows relative positioning by measuring the spatial origin (distance and bearing) of the message being received from another foot-bot.

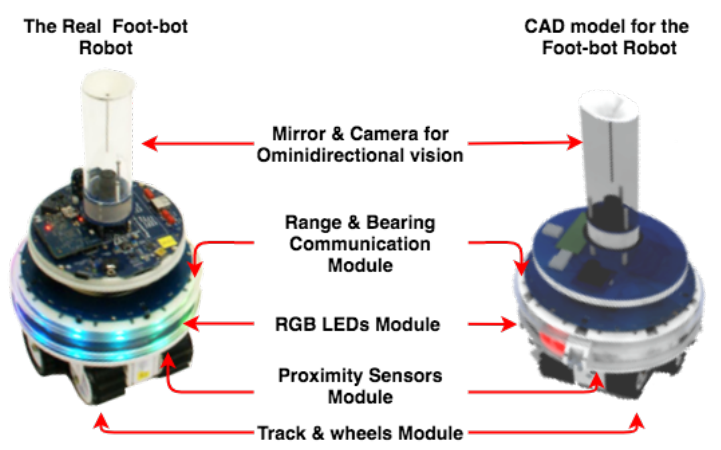

Fig. 1: An overview of the foot-bot robot and its sensors/actuators.

\section{B. Circle Formation Controller with the foot-bots robots}

The idea behind this control model is that it follows an artificial physical approach to emulate inter-robot interactions. This means that robots exert virtual physical forces on one another to achieve the desired circle formation. The physical law used in this study was applied in a number of our previous studies [?], [29], [49]-[51] is based on a virtual viscoelastic force, which can be represented as a spring and damping elements connected in parallel.

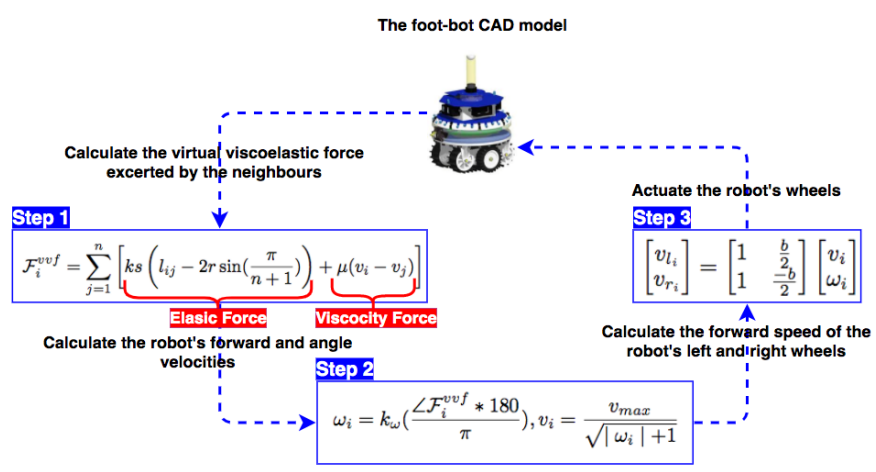

Fig. 2: A schematic description of the virtual viscoelastic based circle formation control model.

More formally, given a circle of a desired radius $r$, to be formed by $N$ foot-bots distributed randomly inside an indoor 
area surrounded by four walls. Then the controller (See Fig.2) implemented on each foot-bot started calculating the total virtual viscoelastic force, $\mathcal{F}_{i}^{v v f}$, exerted by all the sensed neighbors $n$ (Step 1). Two virtual forces per each sensed robot are summed together: the elastic force and the viscosity force to calculate this force. $\mathcal{F}_{i}^{v v f}$ will serve then to actuate the footbot's wheels by calculating first the robot's angular speed, $\omega_{i}$, and its forward speed, $v_{i}$ (Step 2). Then based on the laters, the controller calculates the foot-bot's left and right wheels: $v_{l_{i}}$ and $v_{r_{i}}$ (Step 3). Further details on the descriptions of the different parameters and coefficients values used in the controller can be found in [29].

The main actuators/sensors used to implement the circle formation controller on the foot-bot robot are the IRs RaB sensors and the two-wheel motors. The RaB communication module was explicitly used in step 1 to compute each neighbor's virtual viscoelastic force and communicate velocities between robots. The two-wheel motors are used in step 3 to move the robot. We also incorporate obstacle avoidance controllers using the IRs proximity sensors that are only activated if a robot encounters an obstacle (the walls surrounding the arena, for example).

\section{Measurements \& Data Collection}

Using the open-source ARGoS simulator [30], we implemented the swarm circle formation controller using 8 footbots robots. For both scenarios studies, a final formed circle of radius $1 \mathrm{~m}$ with a robot placed at the center is illustrated in Fig.3. Also, the corresponding trajectories of the foot-bots are plotted in the figure. (a)

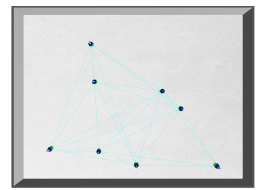

(b)

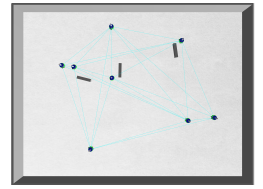

Initial Position
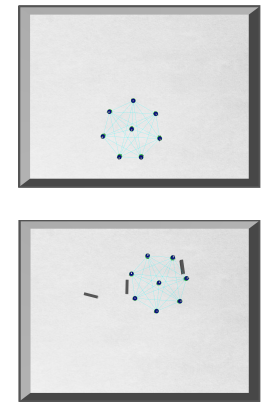

Final Position
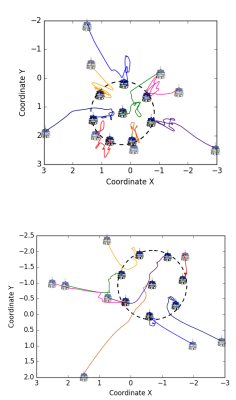

Robots Trajectories
Fig. 3: ARGoS Simulation Results while performing the swarm circle formation controller with 8 foot-bots. (a) the without obstacles scenario and (b) the with obstacles scenario

For a total duration of 150 seconds, we collected measurements every 0.1 second from each foot-bot's sensors/actuators, which have a strict relationship with the inputs/outputs measurements of the implemented controller. Also, measurement that tracks the swarm motion speed, $G S$, of the entire 8 footbots are collected. Note that $100 \mathrm{ARGoS}$ runs in total are performed and the average for each measurement is taken into account. All measurements collected in our study are summarized in Table I.

We collected two datasets for two scenarios: (1) in the absence of obstacles in the arena and (2) in the presence
TABLE I: Measurements Collected Using the ARGoS Simulator while performing the Swarm Circle Formation Controller

\begin{tabular}{ll}
\hline Reading & Description \\
\hline$G S$ & The Swarm Motion Speed \\
$\left.v_{r_{i}}\right|_{i=0} ^{7}$ & The Forward Speed of the Foot-bot's Right Wheel \\
$\left.v_{l_{i}}\right|_{i=0} ^{7}$ & The Forward Speed Foot-bot's Left Wheel \\
$\left.v_{i}\right|_{i=0} ^{7}$ & The Foot-bot Forward Speed \\
$\left.\mathcal{F}_{i}^{v v}\right|_{i=0} ^{7}$ & The Length of the Virtual Viscoelastic Force \\
$\left.\angle \mathcal{F}_{i}^{v v f}\right|_{i=0} ^{7}$ & The angle formed by $\mathcal{F}_{i}^{v v f}$ \\
\hline
\end{tabular}

of obstacles in the arena. Each dataset contains a total of 1500 rows with 41 features. The exploratory data analysis results for the two datasets are provided in Fig.4. The figure shows the overall pairwise correlation matrix between all the features collected for the two scenarios in Fig.4.a and Fig.4.b successively. As shown in the figure in both scenarios, $G S$ is highly positively correlated with $\mathcal{F}_{i}^{v v f}$ and $v_{i}$ and slightly negatively correlated with $\angle \mathcal{F}_{i}^{v v f}, v_{l_{i}}$, and $v_{r_{i}}$. Likewise, $\left.\mathcal{F}_{i}^{v v f}\right|_{i=0} ^{7}$ are almost strongly positively correlated with each other as well as with $G S$ and softly negatively correlated with the remaining features. The results also point out that while there is a high positive correlation between each robot $\angle \mathcal{F}_{i}^{v v f}$ and its $v_{l_{i}}$, the exact opposite correlation is observed between the robot $\angle \mathcal{F}_{i}^{v v f}$ and its $v_{r_{i}}$. However, no linear correlation is observed among the robots pairwise measurements $\angle \mathcal{F}_{i}^{v v f}$. Finally, the robot forward velocity $v_{i}$ is slightly positively correlated with the robot's wheel speeds: $v_{r_{i}}$ and $v_{l_{i}}$, since it can be computed from $v_{r_{i}}$ and $v_{l_{i}}$ as shown in Step 3 of Fig.2.

Measurements data from swarm robotic systems are generally contaminated with noise and uncertainties, and thus relevant features in the measurements may be hidden. This could degrade the data analysis process and the swarm motion speed prediction performance for the chosen ML model. Consequently, in the next subsection, we present the ensemble learning methods used to deal with noise and uncertainties in measurement data.

\section{Ensemble Learning Methods}

Ensemble learning methods are extensive techniques used to enhance ML models performances by combining several learners. In this section, we provide an overview of two commonly used ensemble learning techniques.

1) Boosted Trees (BST): The BST algorithm is a potent predictive model that results from boosting techniques to decision regression/classification tree models [52]. Boosting is a technique that significantly leads to optimizing the prediction accuracy of traditional regression/classification methods by using an adaptive combination of a sequence of relatively simple models [53], [54]. Compared to similar combined techniques such as stacking, bagging, and averaging models, boosting is matchless due to sequentiality [54].

While boosting can be applied to either classification or regression problems, we are interested in using the technique of boosting for a regression problem, where the basic models are regression trees, and the boosting is a form of functional gradient descent algorithm [53]. 

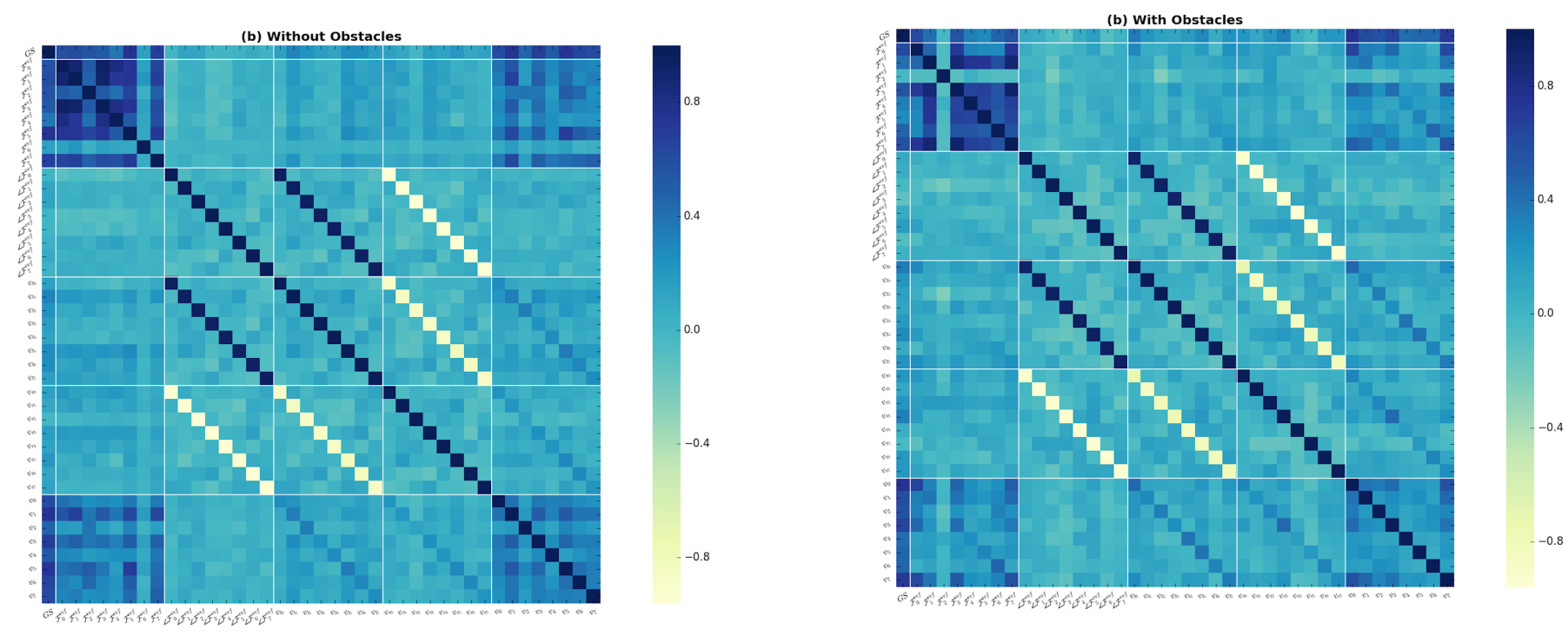

Fig. 4: The Overall Pairwise Correlation Matrixes of all the variables in Table I collected from the ARGoS based Datasets.

To describe the boosted trees algorithm more formally, we shall first provide a brief overview of regression trees. Consider $y \in \mathbb{R}$ is the swarm motion speed and $X \in \mathbb{S} \subset \mathbb{R}^{s}$ is the input features used for predicting the swarm motion speed, where $s$ is the input features number of the feature space $\mathbb{S}$.

Usually in the regression trees algorithm, the feature space $\mathbb{S}$ is divided into a set of $m$ leaves: $\mathbb{S}_{1}, \mathbb{S}_{2}, \ldots, \mathbb{S}_{m}$ representing distinct partitions without overlap. While making predictions with a given tree, the algorithm learns to associate a weight value $w_{i}$ for each leaf $\mathbb{S}_{i}$. The prediction result of an input feature $X \in \mathbb{S}$ is then $w_{i}$, if $X \in \mathbb{S}_{i}$. Note that the weight $w_{i}$ is usually defined as the average of the response variable of $X$ that belongs to $\mathbb{S}_{i}[15]$.

Consider now $\left\{\left(X_{i}, y_{i}\right)_{i=1}^{n}\right\}$ as a given data to be trained with a regression trees algorithm. The feature space $\mathbb{S}$, in this case, is recursively divided into sub-partitions such that the algorithm optimizes an objective function (e.g., the residual sum of squares (RSS)) while satisfying some stopping criterion. Generally speaking, the stopping criterion in the boosting technique can be set to a known number of leaves. For example, a two-leaf regression decision tree implies splitting the feature space $\mathbb{S}$ only once. A more detailed description of the regression tree algorithm can be found in [55].

Theoretically, the variance of prediction using $\mathrm{n}$ learners can be reduced to $1 / \mathrm{n}$ of the original variance (single learner). Thus, the use of many learners is advantageous because it generally results in reduced variance compared to prediction performance obtained small numbers of learners. To understand how the bagging algorithm reduces the mean squared prediction error, let us consider the following regression problem with base regressors $b_{1}(x), \ldots, b_{n}(x)$.

Assume that an ideal target function of true answers $y(x)$ obtained from a given set of inputs is known. Also, suppose that the distribution $p(x)$ is defined. Then, the error is obtained

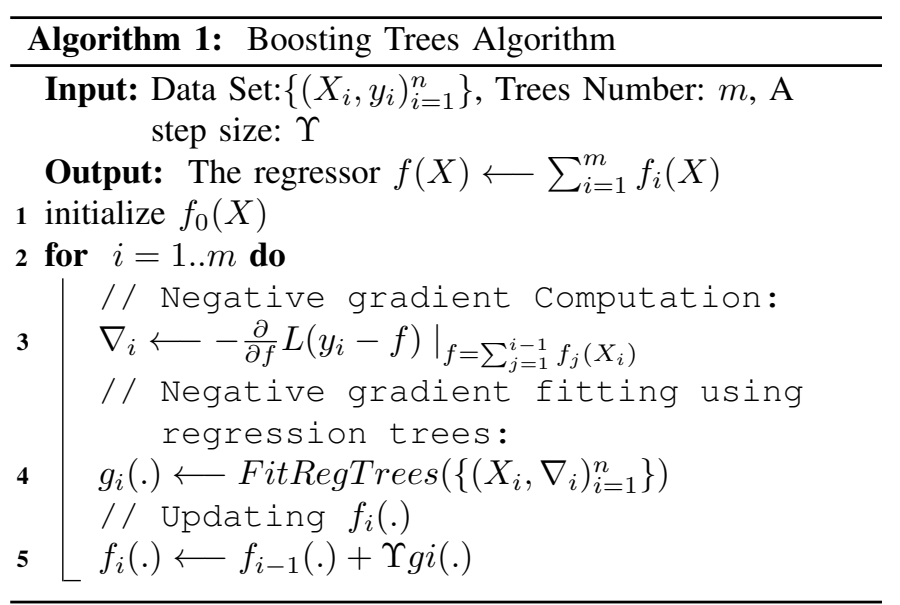

for each regression function as follows:

$$
\varepsilon_{i}(x)=b_{i}(x)-y(x), \quad i=1, \ldots, n
$$

The mean square prediction error has the following form:

$$
\mathbb{E}_{x}\left[\left(b_{i}(x)-y(x)\right)^{2}\right]=\mathbb{E}_{x}\left[\varepsilon_{i}^{2}(x)\right] .
$$

Then, the mean prediction error over all the regression functions is expressed as

$$
\mathbb{E}_{1}=\frac{1}{n} \mathbb{E}_{x}\left[\varepsilon_{i}^{2}(x)\right]
$$

Assume that the errors are unbiased and uncorrelated, then we can write:

$$
\begin{aligned}
\mathbb{E}_{x}\left[\varepsilon_{i}(x)\right] & =0, \\
\mathbb{E}_{x}\left[\varepsilon_{i}(x) \varepsilon_{j}(x)\right] & =0, \quad i \neq j .
\end{aligned}
$$

Using the average of the individual functions, the regression function is calculated as follows:

$$
a(x)=\frac{1}{n} \sum_{i=1}^{n} b_{i}(x) .
$$


Hence, its mean square error is given by

$$
\begin{aligned}
\mathbb{E}_{n} & =\mathbb{E}_{x}\left[\frac{1}{n} \sum_{i=1}^{n} b_{i}(x)-y(x)\right]^{2} \\
& =\mathbb{E}_{x}\left[\frac{1}{n} \sum_{i=1}^{n} \varepsilon_{i}\right]^{2} \\
& =\frac{1}{n^{2}} \mathbb{E}_{x}\left[\sum_{i=1}^{n} \varepsilon_{i}^{2}(x)+\sum_{i \neq j} \varepsilon_{i}(x) \varepsilon_{j}(x)\right] \\
& =\frac{1}{n} \mathbb{E}_{1} .
\end{aligned}
$$

It has been shown above that averaging the individual answers allows us to reduce the mean squared error by a factor of $n$. The main characteristic of bagging is its ability to significantly reduce the variance of prediction error by training the model on different datasets. Hence, bagging has a significant advantage since individual models are trained using different training data, and their prediction errors are gradually reduced during the averaging process. Moreover, outliers' observations are almost removed in some of the training datasets. Sometimes, it can be challenging to interpret the derived model when combining a set of trees in the BTs model. BTs enhances the quality of prediction at a certain expense of interpretability.

Now to apply the boosting algorithm for predicting the swarm motion speed $y$, we consider a function $f^{*}(X)$ such that the error is optimized,

$$
f^{*}(X)=\underset{f(.)}{\arg \min } \mathbb{E}(L(y-f(X))),
$$

where $L($.$) is a loss function usually in the form of the squared$ error loss $L(e)=e^{2}$, and arg min represents the minimum argument minimizing the error on all possible considered functions. To approximate $f^{*}(X)$, the boosting algorithm (See Algorithm 1 and 5 for a brief description) uses a summation function as follows:

$$
f(X)=\sum_{i=1}^{m} f_{i}(X)
$$

such that the $m f_{i}(X)$ stand for regression trees. Note that since the square error loss function is used in the boosting algorithm of this paper, the regression trees are iteratively fitted to the previous fit residuals where the previous errors are gradually corrected by current fits. In addition, at each iteration the resulting fit is slowly moved in the function space by applying a small step size $\Upsilon$.

2) Bagged Trees (BT): BT is another hybrid algorithm that combines the bagging technique and decision trees to enhance prediction accuracy. It has a close relationship with the boosting algorithm in terms of aggregating results of several basic learners [56]. However, the BT algorithm's performance is achieved via building a fixed number $m$ of decision trees, then averaging their performance results together. This considerably reduces the variance of the decision trees and substantially addresses the problem of overfitting a single tree [14], [57]. BT specifically uses bootstrap sampling as an optimization technique to generate $m$ training decision trees and improve their input training data [58]. The technique involves randomly sampling the original training dataset with substitution, which results in replacing the original dataset with $m$ uniform samples used to fit each regression tree.

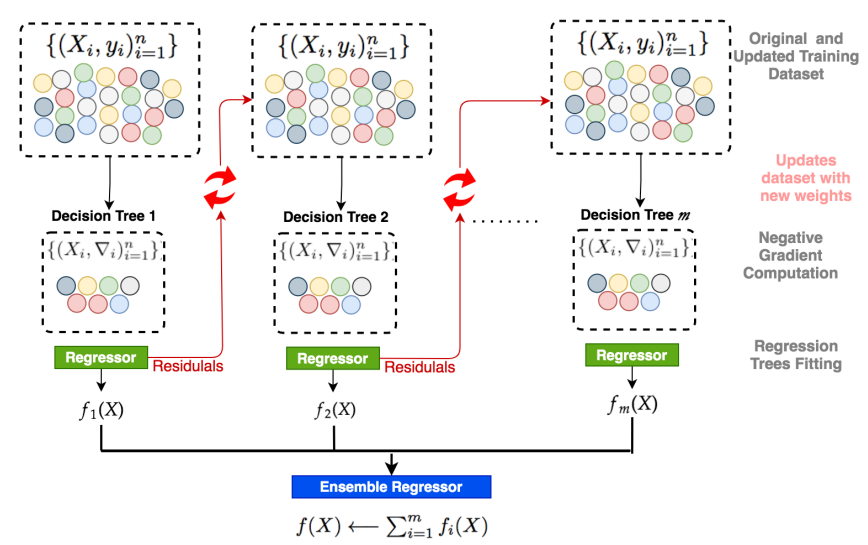

Fig. 5: A Schematic illustration for the concept of BST Algorithm

To describe the BT algorithm more formally, let $\left\{\left(X_{i}, y_{i}\right)_{i=1}^{n}\right\}$ be the entire training data set and $m$ the number of trees to be constituted. At each iteration, a decision tree is trained using various bootstrap samples $\left\{\left(X_{i}^{*}, y_{i}^{*}\right)_{i=1}^{n}\right\}$ selected randomly from the original set of training data. A regression tree is then iteratively fitted to update the final predictor $f(X)$. The main steps of computing the BT algorithm are highlighted in Algorithm 2 and Fig.6.

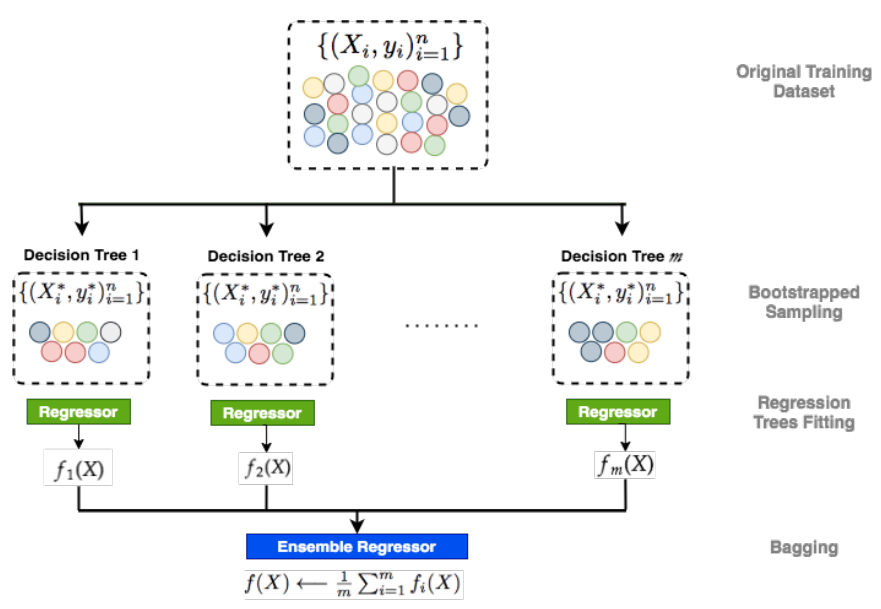

Fig. 6: A Schematic illustration for the concept of BT Algorithm

\section{Prediction Results and Discussion}

\section{A. Models performance evaluation metrics}

To predict the swarm motion speed in our study, we rely on the set of prediction-based regression models summarized in the table of Fig.7, and which are run in three steps, as shown in the left of Fig.7. As a total, thirteen regression models are used to predict the swarm motion speed: ordinary least squares (OLS) regression, six SVR models, four GPR models, and two Ensemble Learning (EL) techniques. SVRs and GPRs are used here to compare the performance of the EL proposed regression models. 


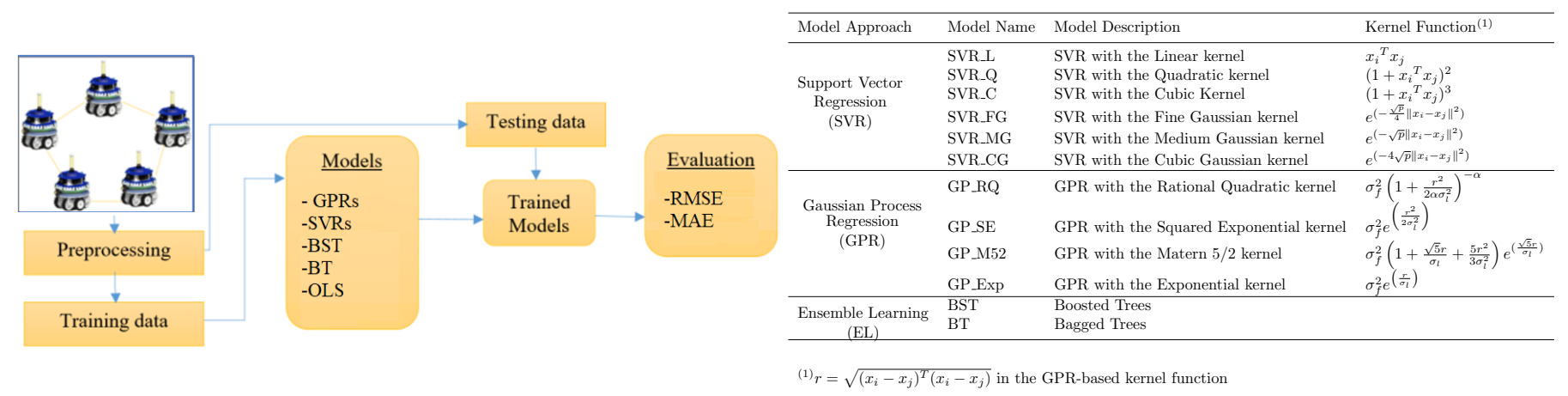

Fig. 7: Regression Models General Data Flow Diagram (left), Regression Models used in our study (right).

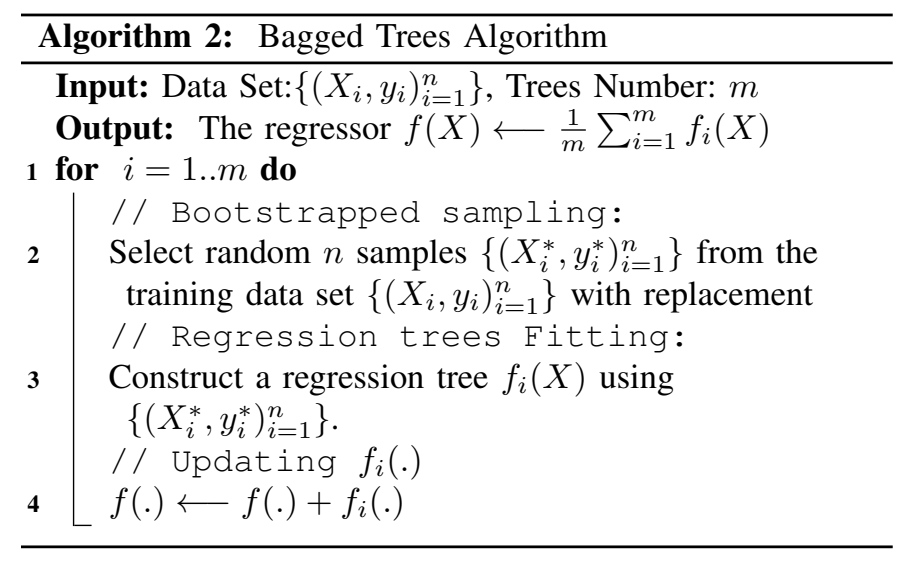

Generally speaking, in a regression prediction model, the correlation between the input features, $X_{i}$, and the corresponding response, $y_{i}$, in the training data is first explored by constructing a regression model. Then to test the model for a given new input data, $\hat{X}_{i}$, the built model will be used to provide the predicted value, $\hat{y}_{i}$. Finally, to check the model performance prediction quality, we use two commonly performance metrics: the root mean squared error, $R M S E$, and the mean absolute error, $M A E$, which can be obtained as follows:

$$
\begin{aligned}
R M S E & =\sqrt{\frac{1}{n} \sum_{i=1}^{n}\left(y_{i}-\hat{y}_{i}\right)^{2}} \\
M A E & =\frac{1}{n} \sum_{i=1}^{n}\left|y_{i}-\hat{y}_{i}\right|
\end{aligned}
$$

Note that the RMSE and MAE values should be minimized for more accurate regression performance, meaning that the lowest values indicate better models. Also, for a proper estimate of the regression prediction error, a frequent technique called the $k$-fold cross-validation (CV) is used while building the regression models [59]. The CV technique is based on a random division of the training dataset into $k$ equal parts (folds). Then, with each iteration, each fold ${ }_{i, i=1 . . k}$ serves as testing data, and the remaining folds serve as a training data set. With this technique, all folds are guaranteed to be used as a testing data set, allowing a reliable regression model to be built. Furthermore, the CV technique uses the cross-validation error, $C V_{e r r}$, as a metric to assess the performance of the built model as follows:

$$
C V_{\text {err }}=\frac{1}{k} \sum_{i=1}^{k} M S E_{i},
$$

where $M S E_{i}$ is the mean square error computed for of each fold used as a testing subset.

Generally, the selection for the appropriate number of folds, $k$, in the CV technique is based on the available dataset's size. Practically, choosing $k=5$ or $k=10$ is widely used in the literature for model building [59]. In this study, 5-fold crossvalidation is used to train the investigated models.

\section{B. Models Construction}

The two datasets that are collected from the ARGoS simulator in Subsection III-C are employed to construct the twelve regression models shown previously in the table of Fig.7. However, not all of the features listed in Subsection III-C are considered to feed the models. To this end, to predict the swarm motion speed, $G S$, we only used $\mathcal{F}_{i}^{v v f}$ and $\angle \mathcal{F}_{i}^{v v f}$ as input features since they are the most important variables contributing to regression models as described in Fig.4 of Subsection III-C. They also represent the only input variables responsible for calculating robot speeds, as illustrated in Fig.2. Note also that $\mathcal{F}_{i}^{v v f}$ has a linear relationship with $G S$ while $\angle \mathcal{F}_{i}^{v v f}$ has a non-linear relationship with $G S$. Our models can then ignore the other features since they provide minimal contributions.

Both datasets are split and normalized into a training dataset $(80 \%)$ and a testing dataset $(20 \%)$. Training datasets are utilized for building regression models. As discussed earlier in this work, twelve regression methods and the OLS method are used to compare the swarm motion speed prediction. Note that in this study and to find the parameters of the models, all the models are fitted based on the CV 5 -folds technique.

The studied models' prediction quality is assessed according to their indicator metrics, namely $R M S E$ and $M A E$. The qualitative results of these metrics when using training datasets are summarized in Fig.8. Results for both case studies scenarios show that the prediction quality of EL-based methods dominate OLS, SVRs, and GPRs in terms of any evaluation metric. Both models score the lowest accuracy in terms of $R M S E$ and $M A E$ metrics. By reaching an accuracy of 


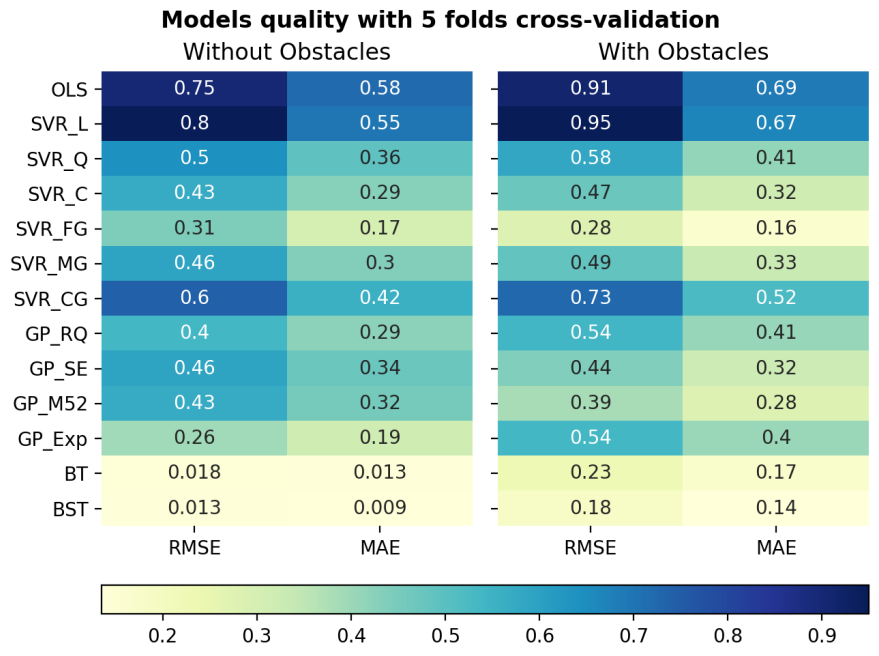

Fig. 8: Predictors Models Quality with 5 Folds Cross-Validation when using training datasets.

$(0.013,0.009)$ and $(0.18,0.14)$ as a $(R M S E, M A E)$ couple for the without and with obstacles scenarios successively, the $B S T$ model shows its superiority to the $B T$ prediction model. The results in Fig. 8 also indicate that the regression performance of OLS and $S V R \_L$ provide relatively poorest performance in both scenarios. These models have only the capacity to extract linear relationships between data features. The according results could be improved as indicated in Fig. 8 when using $S V R$ with different kernels such as the quadratic $(\mathrm{Q})$, the medium gaussian, (MG) the cubic (C), and the cubic gaussian (CG). It is shown that $S V R \_C E$ outperforms OLS and $S V R \_L$. However, better performance results are obtained with the $S V R \_Q$ and the $S V R \_M G$, and the highest performance results are achieved with the $S V R_{-} C$. This due to the ability of the different kernels used in the SVR method to extract non-linear relationships in data features.

On the other hand, applying GPRs approaches may outperform SVRs regression methods to predict swarm motion speed in some scenarios due to their capabilities to discover hidden features relationships better. As shown in Fig.8, the GPR with the exponential (EXP) kernel provides the best results just after the EL-based models in the absence of obstacles scenario. However, using the GPR with the standard exponential (SE) kernel slightly performs at the same level as the $S V R_{-} Q$ and $S V R \_M G$. Similarly, using the GPR with Matern 5/2 (M52) and rational quadratic (RQ) kernels are roughly equal to the performance of $S V R \_C$. These results slightly differ in the obstacle scenario where the SVR with the fine gaussian kernel (FG) provides the best performance compared to the other GPRs and SVRs models.

To confirm the above results, we illustrate via box-plots in Fig.9(a-b) the distribution of models predicted errors, which define the error between the predicted and the true observed values. The most the median of the box-plot tends to zero, the most the model is accurate. As a consequence and as it can be seen from Fig 9, $B T$ and $B S T$ provides better performance than all the other models in both case studies.
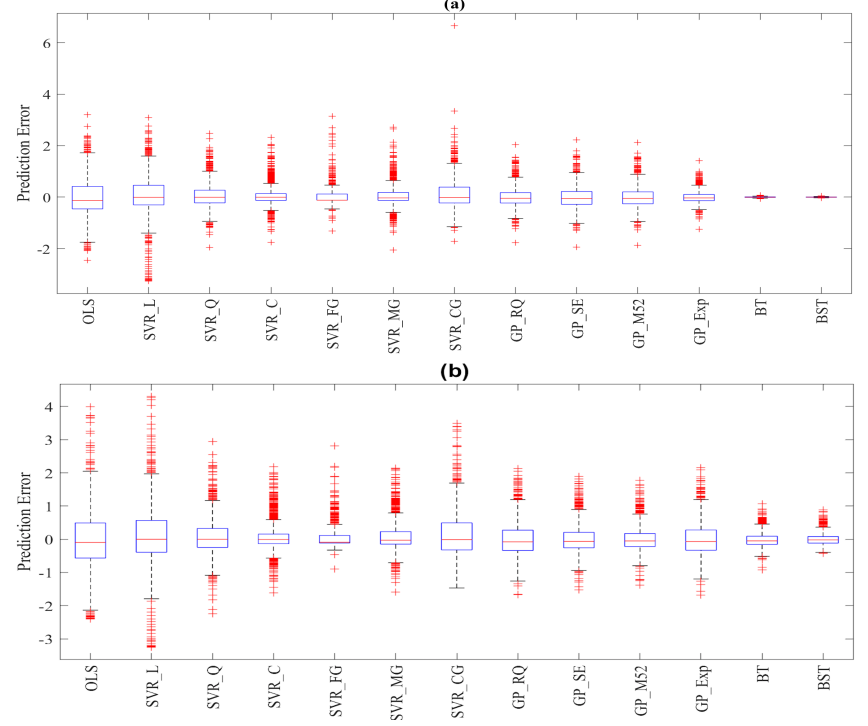

Fig. 9: Models Prediction Errors Results using the training datasets in (a) absence of obstacles and (b) presence of obstacles scenarios.

\section{Prediction Results with a Fully Connected Swarm}

Now we test the built regression models using testing datasets in both scenarios. Figure.10(a-b) illustrates the predicted and measured swarm motion speed using OLS, SVRs, GPRs, and EL-based models. Performance results of the designed models previously optimized using 5-folds CV, when applied to unseen testing samples, are depicted in Fig.11.

It can be seen that for the case study without obstacles, ensemble learning approaches ( $B S T$ and $B T)$ are dominant in terms of both indicator metrics when compared to the Support Vector Regression (SVR) and Gaussian Process Regression (GPR) methods. They both achieve the optimal accuracy in terms of the couple (RMSE, MAE) of about $(0.36,0.27)$ and $(0.3,0.23)$ successively. This is due to the flexibility of $B S T$ and $B T$ as ensemble learning algorithms to capture almost all the important features and patterns existing in the studied dataset and optimize prediction accuracy through models sequencing. The OLS and the $S V R \_L$ models score the worst performance among the other remained models. This fact is due to their inability to discover features in data. On the other hand, using different kernels with SVR provides better performance. The Quadratic SVR $\left(S V R \_Q\right)$ reaches an accuracy of around 0.55 of RMSE, outperforming the Cubic Gaussian, Cubic, and Fine Gaussian SVRs.

The results from Fig.11 highlight also that the predictive accuracy of GPRs built models present a slightly superior performance to SVRs. However, we notice almost an equal performance between the GPRs models and the Matern 5/2 SVR model.

Similarly, the $B S T$ ensemble learning model shows superior performance compared to all the other models for the scenario with obstacles. Specifically, it achieves a precision of around 0.35 for $R M S E$ and 0.28 for $M A E$, which like in the no-obstacles scenario, supports the ability of $B S T$ to optimizing regression accuracy. The other ensemble learning 

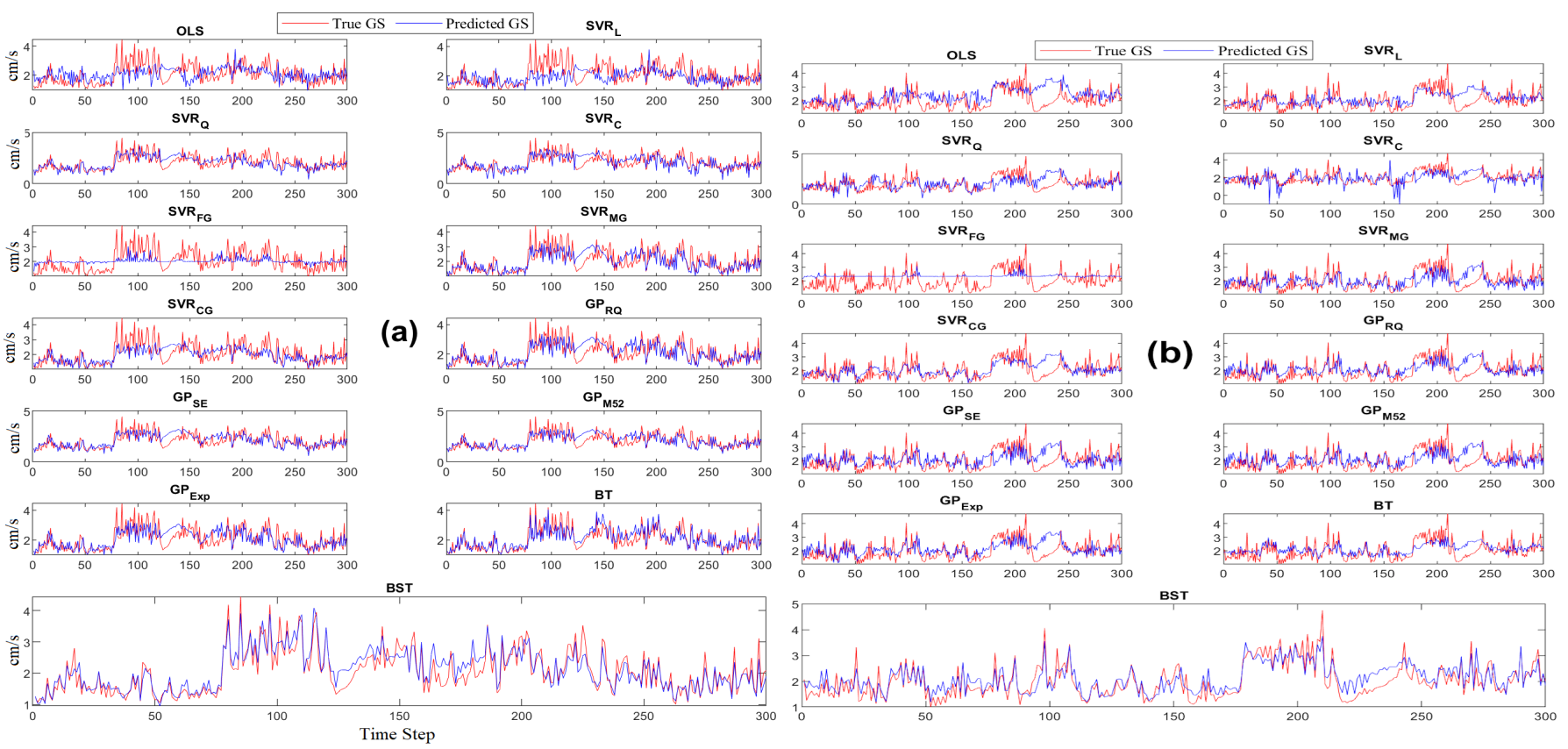

Fig. 10: Comparison of Regression Models Using the Testing Data Sets in both Case Studies Scenarios: (a) without obstacles and (b) with obstacles.

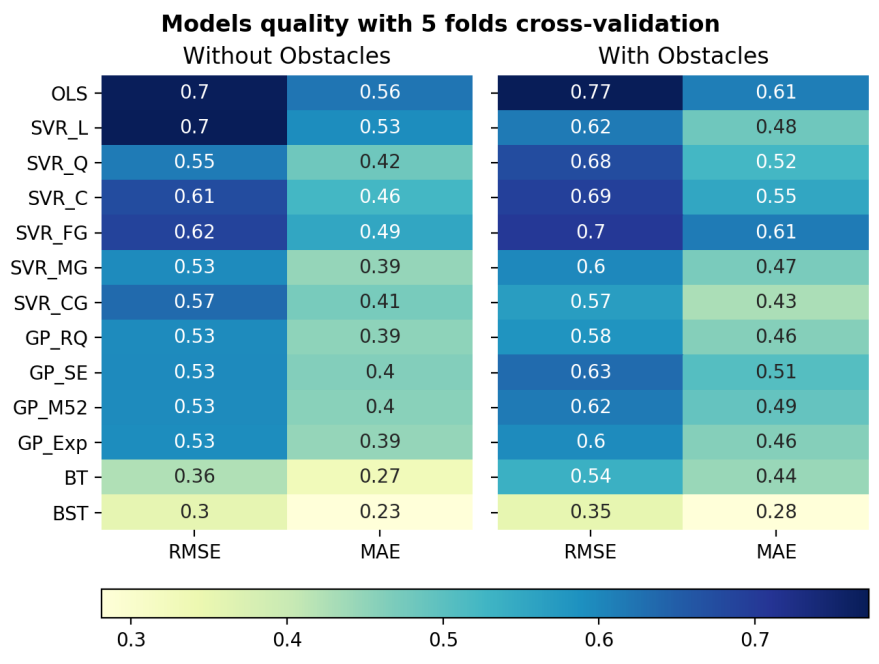

Fig. 11: Predictors Models Quality with 5 Folds Cross-Validation when using testing datasets.

algorithm, $B T$, also provides good results compared to the other built models. It outperforms the OLS, the SVR with linear, Cubic, Quadratic, and Fine Gaussian kernels, and slightly outperforms the GPR models as well as the Medium Gaussian SVR. The last achieve an RMSE accuracy of around 0.6 and performs almost at the same level as the Matern $5 / 2$ GPR, both having the best performances just below $B S T$ and $B T$ models. Results from Fig.11 also show the superiority of almost the other GPRs models when compared to the other SVRs.

This comes down to the inability of these SVRs built-models to discover pertinent features and non-linearity relationships, which in this case study are best discovered by the GPRs models and by the $B T$ model and are even highly discovered using the $B S T$ model. This fact is due to the noise caused by the force exerted by an obstacle on a robot, which has the consequence of disturbing the features $\left(\mathcal{F}_{i}^{v v f}\right.$ and $\left.\angle \mathcal{F}_{i}^{v v f}\right)$ of the robots encountering the obstacle.

Furthermore, we study via boxplots the models' prediction error distribution defined as the difference between the predicted and the observed values cited in equations 9 and 10 . The related results for both studied scenarios are presented in Fig.12.(a) and Fig.12.(b). From Fig.12.(a), we can see that the medians (the red lines in the middle of the boxplots) of the EL models ( $B S T$ and $B T$ ) are almost zero for the without obstacle scenario, which means that $B S T$ and $B T$ provide fewer regression errors than GPRs and SVRs. Consequently, these results reinforce EL models' dominant performance over SVRs or GPRs in predicting the swarm motion speed. Whereas, for the scenario with obstacles shown in Fig.12.(b), it is the median of the $B S T$ algorithm that is almost zero, which confirms its efficiency compared to the other regression models. Besides the box-plots prediction error plots, we also show the error Cumulative Distribution Function (CDF) of the studies models to test their capacity on different prediction steps. Figure 12.(c) and Figure 12.(d) show the according results for both scenarios, and confirm the accurate prediction performance of ensemble models compared to the other prediction models.

\section{Prediction Results with Partially connected Swarm}

In this subsection, we carry out an unusual experiment to identify swarming behaviors and make predictions for the case where the swarm is not fully connected. Crucially, it is essential to see how the proposed prediction approach performs in a swarm with disconnected robots. In other words, 


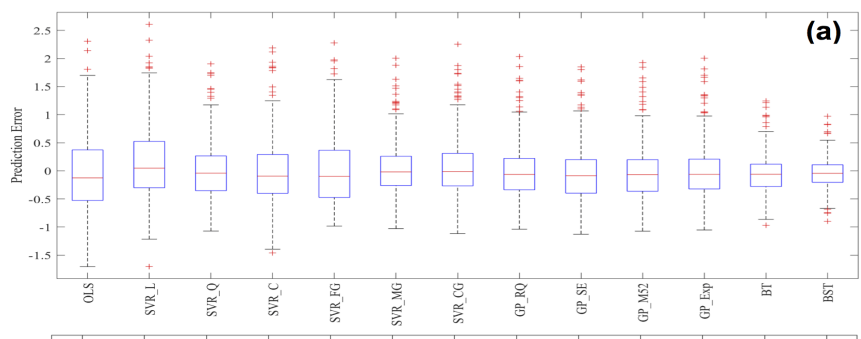

(a)
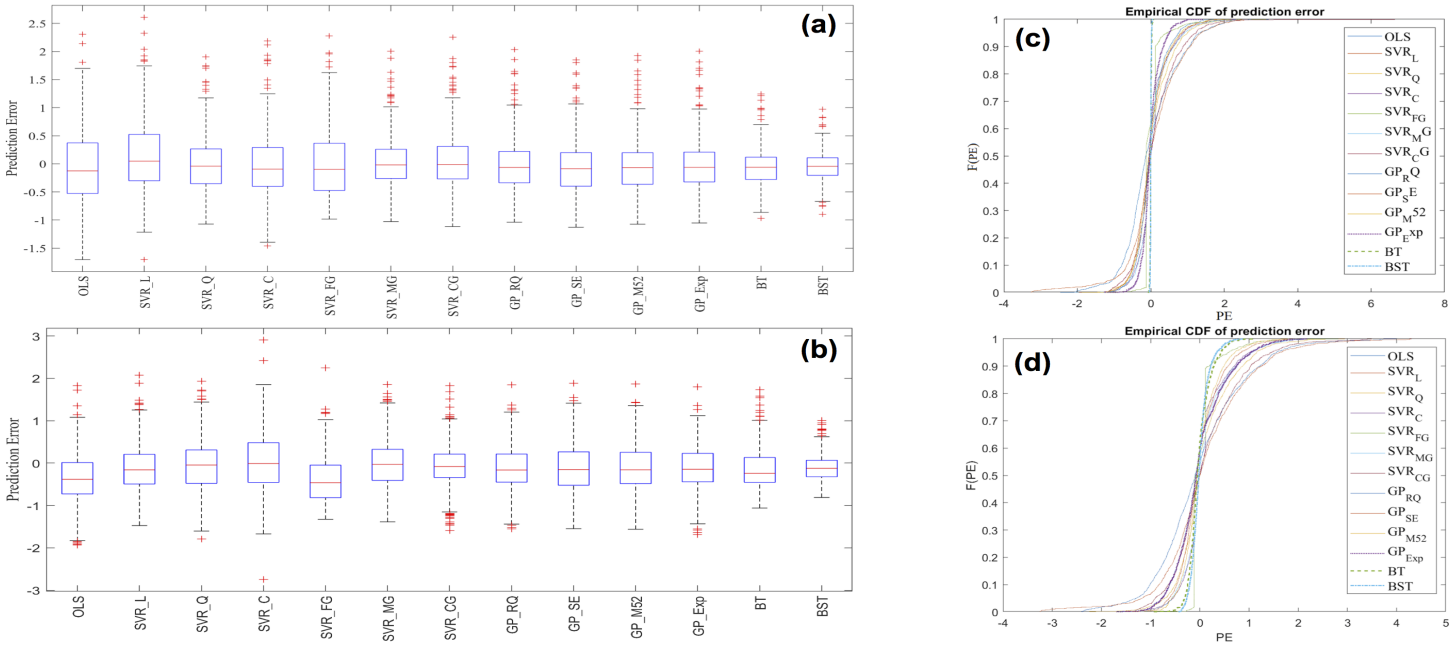

Fig. 12: Models Prediction Errors Results via Box-plots and via Empirical Cumulative Distribution Function (CDF): (a) and (c) are for the without obstacles scenario, (b) and (d) are for the with obstacles scenario.

we are interested in studying the prediction capabilities of the proposed machine learning techniques when the swarm is partially connected. To this end, 100 ARGoS-based simulation runs are conducted for this purpose, and measurements are collected every 0.1 second for a total duration of 150 seconds. Figure 13 shows a scenario where a circle of radius $1 \mathrm{~m}$ is being formed by 7 foot-bots out of 8 robots initially randomly distributed in a non-free obstacles arena. Contrary to the scenarios used in subsection IV-C, the obstacles here totally prevent the foot-bots from sensing one another (See the footbot robot enclosed by a red circle in Fig.13 as an example). This makes the unconnected robot(s) unable to detect all other robots relative to itself, and therefore cannot calculate the centroid's position and state. The measurements collected for this study are the same as in Table I of subsection III-C. As a result, the dataset for this scenario, which is used to construct the regression models as in subsection IV-C, contains a total of 1500 rows with 2 principal features: $\mathcal{F}_{i}^{v v f}$ and $\angle \mathcal{F}_{i}^{v v f}$ out of 41 .

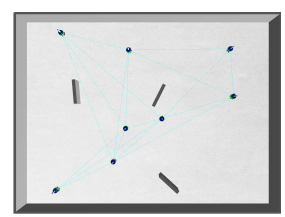

Initial Position

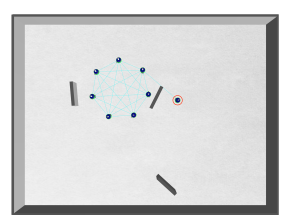

Final Position

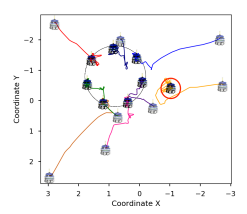

Robots Trajectories
Fig. 13: ARGoS Simulation Results while performing the swarm circle formation controller with 8 foot-bots in presence of obstacles preventing robots to sense neighbours.

The testing prediction results of the built regression models for this scenario, when applied to unseen test samples, are shown in Figure.14. Note that the performance results of the designed models are optimized using 5-fold CV. Results indicate that the ensemble learning approaches $B S T$ and $B T$ utilized in this study outperform the other classical ML regressors in terms of both indicator metrics (RMSE and
$M A E)$. Both achieve an accuracy of around $(0.21,0.16)$ and $(0.45,0.34)$ successively. The $B S T$ build-model provides the highest accuracy when compared to the $B T$ model, which supports the optimizing regression accuracy of $B S T$ (See Fig.15 for the $B S T$ prediction results). In this scenario, although the two principal features used to feed the models are affected by the loss of communications between the robots caused by an obstacle in the robot-robot line-of-sight communication sensors, the prediction performance of the EL-based models (i.e., $B S T$ and $B T$ ) are still dominant. Results again testify the flexibility and power of $B S T$ and $B T$ ensemble learning techniques in seizing almost any significant pattern hidden in data and optimizing prediction accuracy using models sequencing.

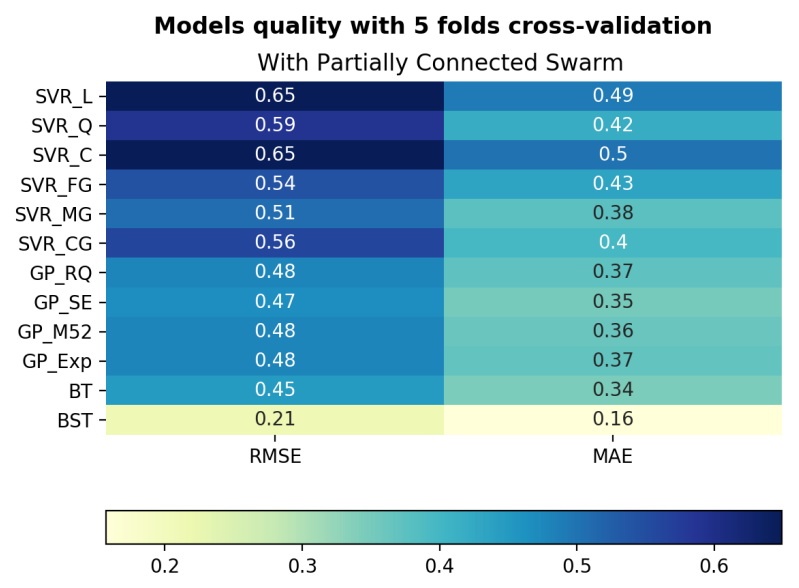

Fig. 14: Predictors Models Quality with Partially Connected Swarm when using testing dataset.

\section{E. Prediction Results with swarm Scalability}

In this subsection, we are interested in studying the robustness of the proposed prediction approach to Swarm Scalability. 


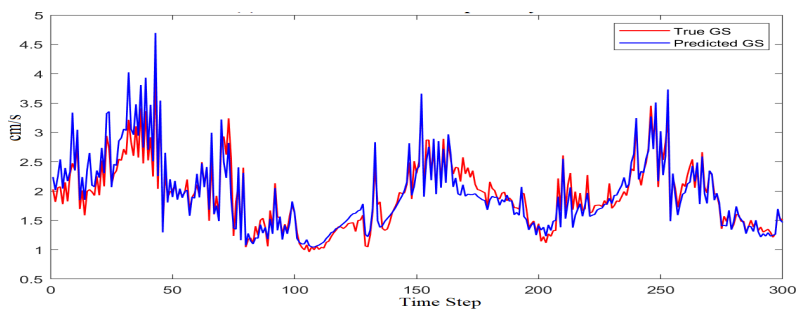

Fig. 15: BST Prediction results with partially connected swarm.

Following the same settings of the previous sections, 100 ARGoS-based simulation experiments are performed with different swarm sizes $N=\{8,12,16,20\}$ in the presence of obstacles preventing the robots from sensing neighbors. The data sets resulting from this scenario were collected for each swarm size case study, then the regression models summarized previously in Table I (subsection III-C) are constructed. In Fig.16, we show the prediction quality results with 5-fold cross-validation when applied the investigated regression models to testing datasets. In this case study, we found that the $B S T$ and $B T$ models are still superior to the other classical ML built models in terms of $R M S E$ and $M A E$ in almost all the swarm size case studies (Fig.16). Also, compared to the classical constructed ML models, we observe that when the swarm size increases, the proposed built-EL regression models' predictive quality increases almost linearly (See the linear decrease in both performance metrics (RMSE and MAE) in the embedded plots). Moreover, the $B S T$ prediction model provides the highest accuracy in both performance metrics in all the swarm size case studies. These suggest that the ELprediction approaches scale linearly with the swarm size. The prediction quality results with different swarm sizes of the best EL-model when applied to the unseen observations are depicted in Fig.17. The results suggest that the $B S T$ model can accurately predict the swarm motion speed $G S$ relative to the true $G S$ for almost all swarm sizes case studies, confirming that our regression EL model can be accurate when the size of the swarm increases.
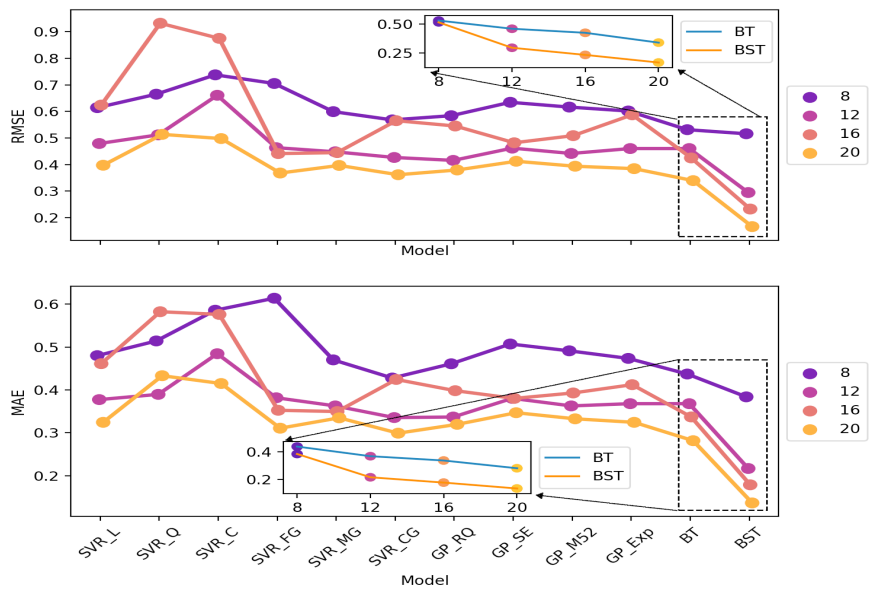

Fig. 16: Predictors Models Quality with different swarm sizes $(N=\{8,12,16,20\}$

) when using the with obstacles testing dataset.

\section{CONCLUSION AND FUtURE WORKS}

In this work, two ensemble learning-based models: Boosted Trees and Bagged Trees, are presented to provide a reliable prediction for a swarm robotics system's motion speed engaged in a circle formation task. To this end, experimental data collected from the sensors/actuators of 8 foot-bots moving to form a circle is applied for model prediction accuracy. Essentially, two datasets addressing two scenarios: in the presence and absence of obstacles in the arena are collected using the ARGoS simulator. In the scenario with obstacles, the swarm is fully connected, meaning that obstacles allow robots to sense each other. Another scenario where the swarm is partially connected was also taken into account. These datasets are used to train the proposed models assessed by two commonly known accuracy prediction metrics: $R M S E$ and $M A E$. The proposed models were also compared to OLS results and SVRs and GPRs models using different kernel functions. Results suggest that the boosted trees and bagged trees regression models provided the highest prediction performance in both scenarios. While it was clearly shown that these two EL-algorithms outperformed all the other models in the without obstacle scenario. However, in the case study with both fully and partially connected swarm, the bagged trees algorithm's prediction performance was slightly better than the cubic Gaussian SVR and the rational quadratic GPR. Moreover, the proposed EL-built predictor approaches' prediction performance quality has been tested using different swarm sizes. Results suggested that with both the Boosted Trees and Bagged Trees models, accurate predictions of the swarm motion speed are achievable when the swarm size increases compared to the classical studied ML models. By using the Boosted Trees and Bagged Trees algorithms as an ensemble learners, we have taken advantage of their ability to provide accurate predictions and greatly minimize the variance of prediction errors to achieve accurate regression performance in predicting swarm motion speed. In addition we have opened a new direction in applying EL predictive modelling techniques as well as other related ML algorithms in the area of swarm robotics research. We believe that this could be a useful tool for model-based monitoring of such robotics systems.

Despite the adequate prediction results of swarm motion speed obtained using ensemble learning techniques, the work presented in this study guides future work. To this end, given an initial condition of the swarm, our approach is tested only to predict the current swarm motion speed. However, it can be enhanced in future work to cope with minimal divergence while the swarm evolves over time. Also, since robot sensor/actuator measurements may contain noisy features that have time and frequency contributions, we plan to enhance the proposed ensemble learning-based swarm motion speed prediction models by developing multi-scale models that combine ensemble learning techniques with statistical quality control charts [60]. It is very interesting also to see how well our predictive built models are accurate when training and testing them in different non-free obstacles environments scenarios. Furthermore, we are planning to use the transfer learning in our future work. Transfer learning, which consists 

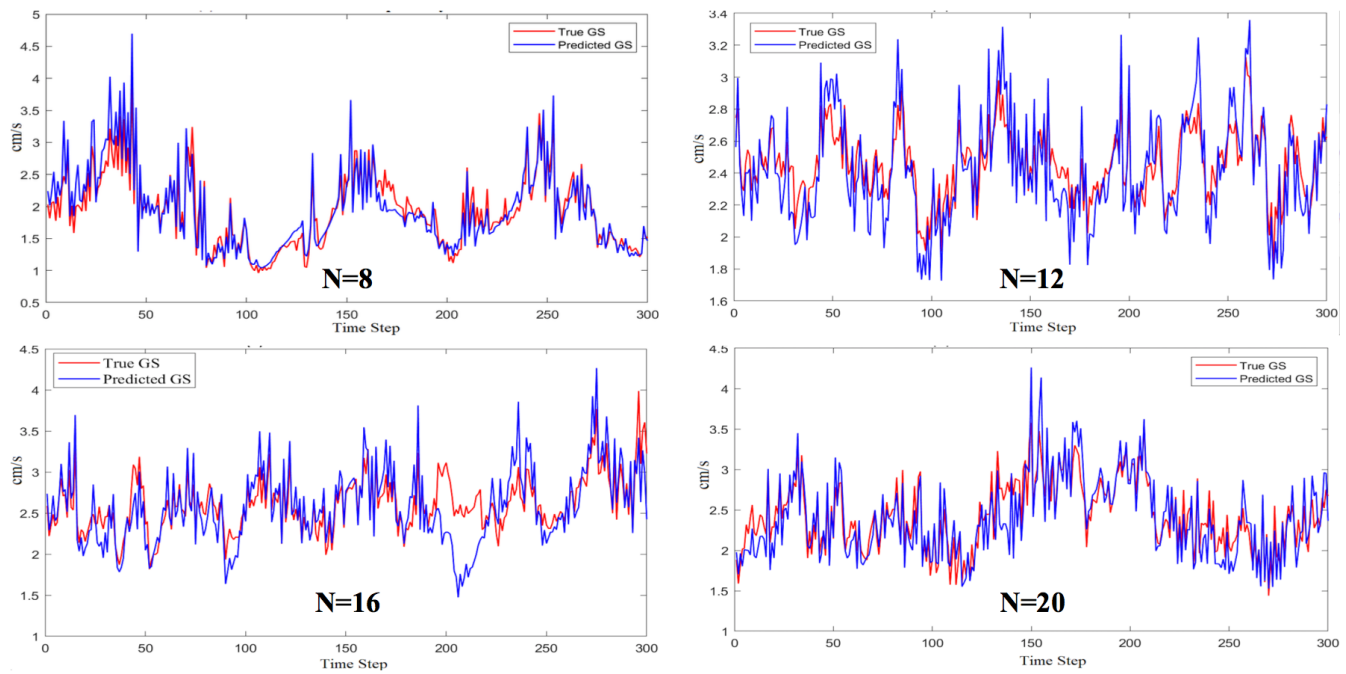

Fig. 17: Predictors Models Quality with different swarm sizes when using the with obstacles testing dataset.

of using the experience obtained by solving predecessor problems (pre-trained models) to improve the process of learning new tasks. This line of research will be investigated in future to improve flexibility of swarm robots. Finally, we plan to design deep learning-based classifiers to discriminate sensors fault and process faults in swarm robots, and a comparison with benchmark classifiers should be investigated. We also aim to use other machine learning prediction techniques in predicting other swarm behaviors, such as aggregation, flocking, task allocation, and collective transportation.

\section{ACKNOWLEDGEMENT}

This publication is based upon a collaboration work from the King Abdullah University of Science and Technology (KAUST) and the Laboratoire de Recherche en Informatique de Sidi Bel-Abbes (LabRI-SBA). The work is supported by KAUST Office of Sponsored Research (OSR) under Award No: OSR-2019-CRG7-3800.

\section{REFERENCES}

[1] E. Şahin, S. Girgin, L. Bayindir, and A. E. Turgut, "Swarm robotics," in Swarm intelligence. Springer, 2008, pp. 87-100.

[2] M. Brambilla, E. Ferrante, M. Birattari, and M. Dorigo, "Swarm robotics: a review from the swarm engineering perspective," Swarm Intelligence, vol. 7, no. 1, pp. 1-41, 2013.

[3] B. Khaldi and F. Cherif, "An overview of swarm robotics: Swarm intelligence applied to multi-robotics," International Journal of Computer Applications, vol. 126, no. 2, 2015.

[4] T. Vicsek and A. Zafeiris, "Collective motion," Physics Reports, vol. 517, no. 3-4, pp. 71-140, 2012.

[5] Y. Tan and Z.-y. Zheng, "Research advance in swarm robotics," Defence Technology, vol. 9, no. 1, pp. 18-39, 2013.

[6] L. Bayındır, "A review of swarm robotics tasks," Neurocomputing, vol. 172, pp. 292-321, 2016.

[7] M. Brambilla, A. Brutschy, M. Dorigo, and M. Birattari, "Propertydriven design for robot swarms: A design method based on prescriptive modeling and model checking," ACM Transactions on Autonomous and Adaptive Systems (TAAS), vol. 9, no. 4, pp. 1-28, 2014.

[8] J. P. Lancaster and D. A. Gustafson, "Predicting the behavior of robotic swarms in search and tag tasks," Procedia Computer Science, vol. 20, pp. 77-82, 2013.

[9] K. Lerman and A. Galstyan, "Two paradigms for the design of artificial collectives," in Collectives and the Design of Complex Systems. Springer, 2004, pp. 231-256.
[10] K. Lerman, M. Mataric, and A. Galstyan, "Mathematical modeling of large multi-agent systems," CALIFORNIA UNIV LOS ANGELES, Tech. Rep., 2005.

[11] J. P. Lancaster Jr, "Predicting the behavior of robotic swarms in discrete simulation," Ph.D. dissertation, Kansas State University, 2015.

[12] H. Hamann, Swarm robotics: A formal approach. Springer, 2018.

[13] L. Pitonakova, R. Crowder, and S. Bullock, "The information-costreward framework for understanding robot swarm foraging," Swarm Intelligence, vol. 12, no. 1, pp. 71-96, 2018.

[14] F. Harrou, A. Saidi, and Y. Sun, "Wind power prediction using bootstrap aggregating trees approach to enabling sustainable wind power integration in a smart grid," Energy Conversion and Management, vol. 201, p. 112077, 2019.

[15] J. Lee, W. Wang, F. Harrou, and Y. Sun, "Wind power prediction using ensemble learning-based models," IEEE Access, vol. 8, pp. $61517-$ $61527,2020$.

[16] A. Soualhi, K. Medjaher, and N. Zerhouni, "Bearing health monitoring based on hilbert-huang transform, support vector machine, and regression," IEEE Transactions on Instrumentation and Measurement, vol. 64, no. 1 , pp. 52-62, 2014.

[17] N. Li, G. Lu, X. Li, and Y. Yan, "Prediction of pollutant emissions of biomass flames through digital imaging, contourlet transform, and support vector regression modeling," IEEE Transactions on Instrumentation and Measurement, vol. 64, no. 9, pp. 2409-2416, 2015.

[18] J. Yoo, K. H. Johansson, and H. J. Kim, "Indoor localization without a prior map by trajectory learning from crowdsourced measurements," IEEE Transactions on Instrumentation and Measurement, vol. 66, no. 11, pp. 2825-2835, 2017.

[19] S. Liu, S. Liu, and T. Ren, "Acoustic tomography reconstruction method for the temperature distribution measurement," IEEE Transactions on Instrumentation and Measurement, vol. 66, no. 8, pp. 1936-1945, 2017.

[20] S. Hazarika, R. Choudhury, B. Montazer, S. Medhi, M. P. Goswami, and U. Sarma, "Detection of citrus tristeza virus in mandarin orange using a customdeveloped electronic nose system," IEEE Transactions on Instrumentation and Measurement, 2020.

[21] D. Dhall, R. Kaur, and M. Juneja, "Machine learning: A review of the algorithms and its applications," in Proceedings of ICRIC 2019. Springer, 2020, pp. 47-63.

[22] B. Khaldi, F. Harrou, F. Cherif, and Y. Sun, "Towards emerging cubic spline patterns with a mobile robotics swarm system," IEEE Transactions on Cognitive and Developmental Systems, 2021.

[23] L. Nesi, D. Antonelli, G. Pepe, and A. Carcaterra, "Fast moving of a population of robots through a complex scenario," in Nonlinear Dynamics and Control. Springer, 2020, pp. 217-225.

[24] B. Khaldi, F. Harrou, F. Cherif, and Y. Sun, "Improving robots swarm aggregation performance through the minkowski distance function," in 2020 6th International Conference on Mechatronics and Robotics Engineering (ICMRE). IEEE, 2020, pp. 87-91.

[25] M. Raoufi, A. E. Turgut, and F. Arvin, "Self-organized collective motion 
with a simulated real robot swarm," in Annual Conference Towards Autonomous Robotic Systems. Springer, 2019, pp. 263-274.

[26] B. Khaldi, F. Harrou, F. Cherif, and Y. Sun, "Flexible and efficient topological approaches for a reliable robots swarm aggregation," IEEE Access, vol. 7, pp. 96372-96383, 2019.

[27] — "Self-organization in aggregating robot swarms: A dw-knn topological approach," Biosystems, vol. 165, pp. 106-121, 2018.

[28] H. Zhao, H. Liu, Y.-W. Leung, and X. Chu, "Self-adaptive collective motion of swarm robots," IEEE Transactions on Automation Science and Engineering, vol. 15, no. 4, pp. 1533-1545, 2018.

[29] B. Khaldi and F. Cherif, "Swarm robots circle formation via a virtual viscoelastic control model," in 2016 8th International Conference on Modelling, Identification and Control (ICMIC). IEEE, 2016, pp. 725730.

[30] C. Pinciroli, V. Trianni, R. O'Grady, G. Pini, A. Brutschy, M. Brambilla N. Mathews, E. Ferrante, G. Di Caro, F. Ducatelle et al., "Argos: a modular, parallel, multi-engine simulator for multi-robot systems," Swarm intelligence, vol. 6, no. 4, pp. 271-295, 2012.

[31] M. Massink, M. Brambilla, D. Latella, M. Dorigo, and M. Birattari, "On the use of bio-pepa for modelling and analysing collective behaviours in swarm robotics," Swarm Intelligence, vol. 7, no. 2-3, pp. 201-228, 2013.

[32] L. Bayindir and E. Sahin, "Modeling self-organized aggregation in swarm robotic systems," in 2009 IEEE Swarm Intelligence Symposium. IEEE, 2009, pp. 88-95.

[33] F. Arvin, S. Watson, A. E. Turgut, J. Espinosa, T. Krajník, and B. Lennox, "Perpetual robot swarm: long-term autonomy of mobile robots using on-the-fly inductive charging," Journal of Intelligent \& Robotic Systems, vol. 92, no. 3-4, pp. 395-412, 2018.

[34] F. Arvin, A. E. Turgut, T. Krajník, S. Rahimi, I. E. Okay, S. Yue, S. Watson, and B. Lennox, " $\phi$ clust: Pheromone-based aggregation for robotic swarms," in 2018 IEEE/RSJ International Conference on Intelligent Robots and Systems (IROS). IEEE, 2018, pp. 4288-4294.

[35] M. Rubenstein, A. Cabrera, J. Werfel, G. Habibi, J. McLurkin, and R. Nagpal, "Collective transport of complex objects by simple robots: theory and experiments," in Proceedings of the 2013 international conference on Autonomous agents and multi-agent systems, 2013, pp. $47-54$.

[36] A. Prorok, N. Correll, and A. Martinoli, "Multi-level spatial modeling for stochastic distributed robotic systems," 2011.

[37] A. Reina, M. Dorigo, and V. Trianni, "Towards a cognitive design pattern for collective decision-making," in International Conference on Swarm Intelligence. Springer, 2014, pp. 194-205.

[38] G. Valentini, H. Hamann, M. Dorigo et al., "Self-organized collective decision making: the weighted voter model." in AAMAS, 2014, pp. 4552 .

[39] G. Valentini, H. Hamann, and M. Dorigo, "Efficient decision-making in a self-organizing robot swarm: On the speed versus accuracy trade-off," in Proceedings of the 2015 International Conference on Autonomous Agents and Multiagent Systems, 2015, pp. 1305-1314.

[40] G. Valentini, E. Ferrante, H. Hamann, and M. Dorigo, "Collective decision with 100 kilobots: Speed versus accuracy in binary discrimination problems," Autonomous Agents and Multi-Agent Systems, vol. 30, no. 3, pp. 553-580, 2016.

[41] H. Hamann, G. Valentini, Y. Khaluf, and M. Dorigo, "Derivation of a micro-macro link for collective decision-making systems," in International Conference on Parallel Problem Solving from Nature. Springer, 2014, pp. 181-190.

[42] D. Goswami and H. Hamann, "Investigation of a collective decision making system of different neighbourhood-size based on hypergeometric distribution," arXiv preprint arXiv:1410.5738, 2014.

[43] J. Zhou, D. Mu, F. Yang, and G. Dai, "A novel approach for analysing collective dynamics of large-scale multi-robot system in task allocation," in 2014 IEEE International Conference on Information and Automation (ICIA). IEEE, 2014, pp. 1137-1142.

[44] J. Guerrero, Ó. Valero, and G. Oliver, "Toward a possibilistic swarm multi-robot task allocation: theoretical and experimental results," Neural Processing Letters, vol. 46, no. 3, pp. 881-897, 2017.

[45] Y. Khaluf, M. Pace, F. Rammig, and M. Dorigo, "Integrals of markov processes with application to swarm robotics modelling," IRIDIA, Université Libre de Bruxelles, Brussels, Belgium, Tech. Rep. TR/IRIDIA/2012-020, 2012.

[46] B. Ranjbar-Sahraei, G. Weiss, and K. Tuyls, "A macroscopic model for multi-robot stigmergic coverage," in Proceedings of the 2013 international conference on Autonomous agents and multi-agent systems. Citeseer, 2013, pp. 1233-1234.
[47] H. Hamann, "Towards swarm calculus: Urn models of collective decisions and universal properties of swarm performance," Swarm Intelligence, vol. 7, no. 2-3, pp. 145-172, 2013.

[48] F. Harrou, B. Khaldi, Y. Sun, and F. Cherif, "An efficient statistical strategy to monitor a robot swarm," IEEE Sensors Journal, vol. 20, no. 4, pp. 2214-2223, 2019.

[49] B. Khaldi, F. Harrou, F. Cherif, and Y. Sun, "Monitoring a robot swarm using a data-driven fault detection approach," Robotics and Autonomous Systems, vol. 97, pp. 193-203, 2017.

[50] B. Khaldi, F. Harrou, Y. Sun, and F. Cherif, "A measurement-based fault detection approach applied to monitor robots swarm," in 2017 6th International Conference on Systems and Control (ICSC). IEEE, 2017, pp. 21-26.

[51] F. Harrou, B. Khaldi, Y. Sun, and F. Cherif, "Monitoring robotic swarm systems under noisy conditions using an effective fault detection strategy," IEEE Sensors Journal, vol. 19, no. 3, pp. 1141-1152, 2018.

[52] Y. Freund and R. E. Schapire, "A decision-theoretic generalization of on-line learning and an application to boosting," Journal of computer and system sciences, vol. 55, no. 1, pp. 119-139, 1997.

[53] J. H. Friedman, "Stochastic gradient boosting," Computational statistics \& data analysis, vol. 38, no. 4, pp. 367-378, 2002.

[54] J. Elith, J. R. Leathwick, and T. Hastie, "A working guide to boosted regression trees," Journal of Animal Ecology, vol. 77, no. 4, pp. 802813,2008

[55] L. Breiman, J. Friedman, C. J. Stone, and R. A. Olshen, Classification and regression trees. CRC press, 1984.

[56] Y. Zhang and A. Haghani, "A gradient boosting method to improve travel time prediction," Transportation Research Part C: Emerging Technologies, vol. 58, pp. 308-324, 2015.

[57] M. D. C. Ruiz-Abellón, A. Gabaldón, and A. Guillamón, "Load forecasting for a campus university using ensemble methods based on regression trees," Energies, vol. 11, no. 8, p. 2038, 2018.

[58] L. Breiman, "Bagging predictors," Machine learning, vol. 24, no. 2, pp. 123-140, 1996.

[59] T. Hastie, R. Tibshirani, and J. Friedman, The elements of statistical learning: data mining, inference, and prediction. Springer Science \& Business Media, 2009.

[60] F. Harrou, Y. Sun, A. S. Hering, M. Madakyaru et al., Statistical process monitoring using advanced data-driven and deep learning approaches: theory and practical applications. Elsevier, 2020. 